\title{
THE RIGID ELLIPSOID IN THE PRESENCE OF A LOW FREQUENCY ELASTIC WAVE*
}

\author{
BY \\ GEORGE DASSIOS (University of Patras) \\ AND \\ KIRIAKIE KIRIAKI ( National Technical University of Athens)
}

\begin{abstract}
A longitudinal, or a transverse, plane elastic wave is incident on a rigid triaxial ellipsoid. The zeroth-order and first-order low-frequency approximations are obtained explicitly at every point exterior to the ellipsoid by solving appropriate exterior boundary value problems of potential theory. The normalized scattering amplitudes are evaluated up to the $k^{2}$-order and the leading term of the scattering cross section is given explicitly.

Corresponding results for the prolate and the oblate spheroid, the needle, the disc, and the sphere are obtained as degenerate ellipsoids.

The calculations were made possible by introducing a fictitious scalar Papkovich-Grodski potential which is appropriately chosen in every case.
\end{abstract}

1. Introduction. In a previous paper [7] we have presented the theory of scattering for the case of a low-frequency elastic wave incident upon a rigid scatterer, or a cavity. The incident wave could be either a longitudinal or a transverse wave. We have managed to reduce the scattering problem into an iterative process that involves the evaluation of certain surface integrals and the solution of an exterior potential problem. Of course, the solutions of the above potential problems are by no means easy to obtain. Nevertheless they are much simpler than the corresponding wave problem which in almost all cases is impossible to solve.

The scattering of a longitudinal wave by a sphere was investigated for the first time by Ying and Truell [16]. Einspruch, Witterholt and Truell [9] have also solved the corresponding problem for transverse incidence. Lawrence [12] used an analytical technique to evaluate the leading low-frequency term for the scattering cross section of an ellipsoid. His work was based on the work of Barratt and Collins [1] for the scattering cross section for a general obstacle. More work for scattering in ellipsoidal geometry can be found in [2-6, 8] while for the fundamental scattering theorems we refer to [14].

* Received May 4, 1984. This work constitutes a part of the doctoral dissertation of the second author performed under the guidance of the first. 
The purpose of this work is to explore, in a systematic way, the low-frequency scattering problems for (i) a longitudinal and (ii) a transverse plane wave incident upon a rigid triaxial ellipsoid, using the analytical technique developed in our previous paper [7].

In Sec. 2 we give the formulation of the scattering problems encountered and provide, for the sake of completeness, the necessary results from [7] without any quantitative analysis.

Section 3 is the key section to our work. Our analytical method is now applied to the ellipsoidal geometry reducing the potential problems involved to the evaluation of the coefficients of certain finite eigenfunction expansions. This is achieved by introducing a fictitious scalar Papkovich-Grodski [13, 15] potential and using its dependence on the corresponding vector potentials to reduce all the complete eigenfunction expansions to finite sums. The method of solution described in Sec. 3 is applicable to the determination of all low-frequency fields, but, as expected, the calculational efforts increase very rapidly with the order of the approximation field.

The zeroth-order low-frequency field is evaluated explicitly in Sec. 4 by using the method of Sec. 3 for both longitudinal and transverse incidence at the same time. Much more work has to be done in order to evaluate the first-order field. The results of these long and tedious calculations are given in Sec. 5. Many relations involving the constants of the ellipsoidal harmonics as well as relations among elliptic integrals had to be worked out in order to bring the final result in the given form. Transformations from the Cartesian to the ellipsoidal system and vice versa were also found and used extensively. Finally the expression of the appearing integrands in terms of surface ellipsoidal harmonics and the evaluation of the relative integrals was another difficulty that had to be overcome.

The final results, both for the zeroth-order and the first-order low-frequency fields are given in terms of expressions involving second and fourth rank tensors dependent on the geometry of the scatterer, the physics of the problem, and the ellipsoidal variable that determines the distance of the observation point from the scatterer. The direction of incidence and the observation point appear explicitly through simple and multiple contractions with the above mentioned tensors. In the given form the dependence of the low-frequency fields on the relative variables of the problem becomes as explicit as possible. A comparison of the zeroth-order with the first-order field gives a measure of the increasing difficulty in the evaluation of the higher-order low-frequency fields.

Section 6 is devoted to the evaluation of the normalized spherical scattering amplitudes and the scattering cross section. This task is reduced to the calculation of certain surface integrals over the surface of the scatterer, which in turn are evaluated by using a combination of spherical and ellipsoidal coordinates. The first nonvanishing term of the scattering amplitudes is proportional to the first power of the wave number, while for the scattering cross section the first term is independent of the wave number.

The special geometrical cases of the prolate and the oblate spheroid, the needle, the disc, and the sphere are discussed in Sec. 7.

Ending this introduction we want to emphasize the many difficulties that appeared in every step of this work and the extremely long calculations that had to be done, which of course are not included in these pages. These difficulties and complications reflect the lack of symmetry of the general ellipsoidal geometry. 
2. Formulation of the problem. Let us assume that the solid triaxial ellipsoid

$$
\sum_{i=1}^{3} \frac{x_{i}^{2}}{\alpha_{i}^{2}} \leqq 1, \quad 0<\alpha_{3}<\alpha_{2}<\alpha_{1}<+\infty,
$$

is embedded in an infinite, homogeneous, and isotropic elastic medium that fills up the complement $V$ of the solid ellipsoid (1) in $R^{3}$. The elastic medium is characterized by the Lamé constants $\lambda, \bar{\mu}$ and the normalized, by scaling, mass density $\rho=1$. The boundary of the ellipsoid (1) is denoted by $S$.

A harmonic time dependence $\exp \{-i \omega t\}$, where $\omega$ is the angular frequency, is suppressed throughout this work.

An "incident wave" $\Phi(\mathbf{r})$ propagates in the elastic medium $V$ along the propagation vector $\mathbf{k}$. The incident wave could be either a plane longitudinal $(P)$ wave

$$
\boldsymbol{\Phi}^{p}(\mathbf{r})=\hat{\mathbf{k}} e^{i k_{p} \hat{\mathbf{k}} \cdot \mathbf{r}},
$$

which is polarized along the direction of propagation $\hat{\mathbf{k}}=\left(i_{1}, i_{2}, i_{3}\right)$, or a plane transverse $(S)$ wave

$$
\Phi^{s}(\mathbf{r})=\hat{\mathbf{b}} e^{i k, \hat{\mathbf{k}} \cdot \mathbf{r}},
$$

which is polarized in the direction $\hat{\mathbf{b}}=\left(b_{1}, b_{2}, b_{3}\right)$ such that $\hat{\mathbf{b}} \cdot \hat{\mathbf{k}}=0$. The wave numbers $k_{p}$ and $k_{s}$ are related to the corresponding wavelengths $\lambda_{p}$ and $\lambda_{s}$ by the expressions $k_{p}=2 \pi / \lambda_{p}$ and $k_{s}=2 \pi / \lambda_{s}$.

The ellipsoid (1), called "the scatterer", disturbs the propagation of the incident wave $\boldsymbol{\Phi}(\mathbf{r})$ in $V$ and let $\mathbf{u}(\mathbf{r})$ be the wave field that describes this disturbance. The total wave field $\boldsymbol{\Psi}(\mathbf{r})$ in $V$ is then (due to linearity) given by the sum of the incident field $\Phi(\mathbf{r})$ plus the "scattered field" u(r), i.e.,

$$
\boldsymbol{\Psi}(\mathbf{r})=\boldsymbol{\Phi}(\mathbf{r})+\mathbf{u}(\mathbf{r}), \quad \mathbf{r} \in V .
$$

The scattered field $\mathbf{u}(\mathbf{r})$, as well as the incident field $\Phi(\mathbf{r})$ and therefore the total field $\Psi$ too, satisfy the time-independent linearized equation of dynamic elasticity

$$
\bar{\mu} \Delta \mathbf{u}+(\lambda+\bar{\mu}) \nabla(\nabla \cdot \mathbf{u})+\omega^{2} \mathbf{u}=\mathbf{0}
$$

at every $\mathbf{r} \in V$.

Using Poisson's decomposition, the scattered field is written as

$$
\mathbf{u}(\mathbf{r})=\mathbf{u}^{p}(\mathbf{r})+\mathbf{u}^{s}(\mathbf{r}), \quad \mathbf{r} \in V,
$$

where $\mathbf{u}^{p}(\mathbf{r})$ and $\mathbf{u}^{s}(\mathbf{r})$ represent the longitudinal and the transverse parts of $\mathbf{u}(\mathbf{r})$, respectively.

Since the ellipsoid (1) is rigid, the boundary condition on $S$ is

$$
\boldsymbol{\Psi}(\mathbf{r})=\mathbf{0}, \quad \mathbf{r} \in S .
$$

The longitudinal and transverse parts of the scattered field satisfy the following radiation conditions which are due to Kupradze [11]:

$$
\begin{array}{ll}
\lim _{r \rightarrow+\infty} \mathbf{u}^{p}(\mathbf{r})=0, & \lim _{r \rightarrow+\infty}\left[\partial \mathrm{u}^{p}(\mathbf{r})-i k_{p} \mathbf{u}^{p}(\mathbf{r})\right] r=\mathbf{0}, \\
\lim _{r \rightarrow+\infty} \mathbf{u}^{s}(\mathbf{r})=0, & \lim _{r \rightarrow+\infty}\left[\partial, \mathbf{u}^{s}(\mathbf{r})-i k_{s} \mathbf{u}^{s}(\mathbf{r})\right] r=\mathbf{0},
\end{array}
$$

where the convergence is uniform over all directions. 
In [7] we have shown that the solution of the above problem has the integral representation

$$
\boldsymbol{\Psi}(\mathbf{r})=\boldsymbol{\Phi}(\mathbf{r})-\frac{1}{4 \pi} \int_{s} \tilde{\Gamma}\left(\mathbf{r}, \mathbf{r}^{\prime}\right) \cdot T_{\mathbf{r}^{\prime}} \boldsymbol{\Psi}\left(\mathbf{r}^{\prime}\right) d s\left(\mathbf{r}^{\prime}\right) .
$$

The tensor $\tilde{\Gamma}\left(\mathbf{r}, \mathbf{r}^{\prime}\right)$ is the fundamental dyadic which satisfies the tensor form of the stationary Navier equation

$$
\left[c_{s}^{2} \tilde{\mathbf{I}} \Delta_{\mathbf{r}^{\prime}}+\left(c_{p}^{2}-c_{s}^{2}\right) \nabla_{\mathbf{r}^{\prime}} \otimes \nabla_{\mathbf{r}^{\prime}}+\omega^{2} \tilde{\mathbf{I}}\right] \cdot \tilde{\Gamma}\left(\mathbf{r}, \mathbf{r}^{\prime}\right)=-4 \pi \delta\left(\mathbf{r}-\mathbf{r}^{\prime}\right) \tilde{\mathbf{I}}
$$

where $\delta$ is the Dirac measure concentrated at $\mathbf{r}, \tilde{\mathrm{I}}=\hat{\mathbf{x}}_{1} \otimes \hat{\mathbf{x}}_{1}+\hat{\mathbf{x}}_{2} \otimes \hat{\mathbf{x}}_{2}+\hat{\mathbf{x}}_{3} \otimes \hat{\mathbf{x}}_{3}$ is the identity dyadic and $c_{p}, c_{s}$ are the phase velocities of the longitudinal and the transverse waves, respectively, given by

$$
c_{p}=\sqrt{\lambda+2 \bar{\mu}}, \quad c_{s}=\sqrt{\bar{\mu}} .
$$

The analytical form of $\tilde{\Gamma}\left(\mathbf{r}, \mathbf{r}^{\prime}\right)$ is given by

$$
\begin{aligned}
\tilde{\Gamma}\left(\mathbf{r}, \mathbf{r}^{\prime}\right)= & \frac{k_{s}^{2}}{\omega^{2}}\left|\mathbf{r}-\mathbf{r}^{\prime}\right|^{-1} \exp \left\{i k_{s}\left|\mathbf{r}-\mathbf{r}^{\prime}\right|\right\} \tilde{\mathbf{I}} \\
& -\frac{1}{\omega^{2}} \nabla \otimes \nabla\left[\left|\mathbf{r}-\mathbf{r}^{\prime}\right|^{-1} \exp \left\{i k_{p}\left|\mathbf{r}-\mathbf{r}^{\prime}\right|\right\}\right] \\
& +\frac{1}{\omega^{2}} \nabla \otimes \nabla\left[\left|\mathbf{r}-\mathbf{r}^{\prime}\right|^{-1} \exp \left\{i k_{s}\left|\mathbf{r}-\mathbf{r}^{\prime}\right|\right\}\right] .
\end{aligned}
$$

The operator

$$
T_{\mathbf{r}^{\prime}}=2 \bar{\mu} \hat{\mathbf{n}}^{\prime} \cdot \nabla_{\mathbf{r}^{\prime}}+\lambda \hat{\mathbf{n}}^{\prime} \operatorname{div}_{\mathbf{r}^{\prime}}+\bar{\mu} \hat{\mathbf{n}}^{\prime} \times \operatorname{rot}_{\mathbf{r}^{\prime}}
$$

describes the surface traction on the scattering surface $S$, where $\hat{\mathbf{n}}^{\prime}$ is the exterior unit normal on $S$.

We have also proved in [7] that the normalized spherical scattering amplitudes are given by the expressions

$$
\begin{aligned}
& g_{r}(\hat{\mathbf{r}}, \hat{\mathbf{k}})=-\frac{i k_{p}}{c_{p}^{2}}\left(\mathbf{I}_{p} \cdot \hat{\mathbf{r}}\right), \\
& g_{\vartheta}(\hat{\mathbf{r}}, \hat{\mathbf{k}})=-\frac{i k_{s}}{c_{s}^{2}}\left(\mathbf{I}_{s} \cdot \hat{\boldsymbol{\vartheta}}\right), \\
& g_{\varphi}(\hat{\mathbf{r}}, \hat{\mathbf{k}})=-\frac{i k_{s}}{c_{s}^{2}}\left(\mathbf{I}_{s} \cdot \hat{\varphi}\right)
\end{aligned}
$$

where

$$
\begin{aligned}
& \mathbf{I}_{p}=\frac{1}{4 \pi} \int_{S} \exp \left\{-i k_{p} \hat{\mathbf{r}} \cdot \mathbf{r}^{\prime}\right\} T_{\mathbf{r}^{\prime}} \Psi\left(\mathbf{r}^{\prime}\right) d s\left(\mathbf{r}^{\prime}\right), \\
& \mathbf{I}_{s}=\frac{1}{4 \pi} \int_{S} \exp \left\{-i k_{s} \hat{\mathbf{r}} \cdot \mathbf{r}^{\prime}\right\} T_{\mathbf{r}^{\prime}} \boldsymbol{\Psi}\left(\mathbf{r}^{\prime}\right) d s\left(\mathbf{r}^{\prime}\right) .
\end{aligned}
$$

The scattering cross section, which is actually a measure of the interaction of the scattered and the incident wave, is defined as the ratio of the time average rate at which 
energy is scattered by the body, to the corresponding time average rate at which the energy of the incident wave crosses a unit area normal to the direction of propagation. The scattering cross sections $\sigma^{p}$ and $\sigma^{s}$, corresponding to longitudinal and transverse incidence, respectively, are given [7] by the expressions

$$
\begin{aligned}
& \sigma^{p}=k_{p} \int_{|\hat{\mathbf{r}}|=1}\left[k_{p}^{-3}\left|g_{r}^{p}\right|^{2}+k_{s}^{-3}\left(\left|g_{\theta}^{p}\right|^{2}+\left|g_{\varphi}^{p}\right|^{2}\right)\right] d \Omega(\hat{\mathbf{r}}), \\
& \sigma^{s}=k_{s} \int_{|\hat{\mathbf{r}}|=1}\left[k_{p}^{-3}\left|g_{r}^{s}\right|^{2}+k_{s}^{-3}\left(\left|g_{\theta}^{s}\right|^{2}+\left|g_{\varphi}^{s}\right|^{2}\right)\right] d \Omega(\hat{\mathbf{r}}),
\end{aligned}
$$

where $g_{r}^{p}, g_{\theta}, g_{\varphi}^{p}$ are the normalized spherical scattering amplitudes that come out of a longitudinal incident wave and $g_{r}^{s}, g_{\theta}^{s}, g_{\varphi}^{s}$ are the corresponding amplitudes for a transverse incident wave.

In [7] we have shown that if we consider the low-frequency expansion

$$
\Psi(\mathbf{r})=\sum_{n=0}^{\infty} \frac{(i \tau k)^{n}}{n !} \boldsymbol{\Phi}_{n}(\mathbf{r}), \quad \mathbf{r} \in V,
$$

where we use the parameters

$$
\left.\tau=\frac{k_{p}}{k_{s}}=\frac{c_{s}}{c_{p}}=\sqrt{\frac{\bar{\mu}}{\lambda+2 \bar{\mu}}}\right\} ;
$$

then for every $n=0,1,2, \ldots$, the coefficient $\Phi_{n}(\mathbf{r})$, which forms the $n$th order lowfrequency approximation of our scattering problem, is given by the sum

$$
\Phi_{n}(\mathbf{r})=\mathbf{P}_{n}(\mathbf{r})+\mathbf{U}_{n}(\mathbf{r}) .
$$

The function $\mathbf{P}_{n}(\mathbf{r})$ is given by

$$
\mathbf{P}_{n}(\mathbf{r})=\pi^{n}(\hat{\mathbf{k}} \cdot \mathbf{r})^{n}-\frac{1}{4 \pi \bar{\mu}} \sum_{\rho=0}^{n-1}\left(\begin{array}{l}
n \\
\rho
\end{array}\right) \int_{S^{\prime}} \tilde{\gamma}_{n-\rho}\left(\mathbf{r}, \mathbf{r}^{\prime}\right) \cdot T_{\mathbf{r}^{\prime}} \mathbf{\Phi}_{\rho}\left(\mathbf{r}^{\prime}\right) d s\left(\mathbf{r}^{\prime}\right)
$$

where

$$
\pi^{n}= \begin{cases}\hat{\mathbf{k}}, & \text { when } \boldsymbol{\Phi}=\boldsymbol{\Phi}^{p} \\ \hat{\mathbf{b}} / \tau^{n}, & \text { when } \boldsymbol{\Phi}=\boldsymbol{\Phi}^{s}\end{cases}
$$

and

$$
\begin{aligned}
\tilde{\gamma}_{n}\left(\mathbf{r}, \mathbf{r}^{\prime}\right)=\frac{\left|\mathbf{r}-\mathbf{r}^{\prime}\right|^{n-1}}{\tau^{n}}\left\{\left[1+\frac{\tau^{n+2}-1}{n+2}\right] \tilde{\mathrm{I}}\right. \\
\left.+(n-1) \frac{\tau^{n+2}-1}{n+2} \frac{\left(\mathbf{r}-\mathbf{r}^{\prime}\right) \otimes\left(\mathbf{r}-\mathbf{r}^{\prime}\right)}{\left|\mathbf{r}-\mathbf{r}^{\prime}\right|^{2}}\right\}
\end{aligned}
$$

is the $n$th coefficient of the low-frequency expansion of the fundamental tensor $\tilde{\Gamma}\left(\mathbf{r}, \mathbf{r}^{\prime}\right)$ indicated by

$$
\tilde{\Gamma}\left(\mathbf{r}, \mathbf{r}^{\prime}\right)=\frac{1}{\bar{\mu}} \sum_{n=0}^{\infty} \frac{(i \tau k)^{n}}{n !} \tilde{\gamma}_{n}\left(\mathbf{r}, \mathbf{r}^{\prime}\right)
$$


The function $\mathbf{U}_{n}(\mathbf{r})$ in (24) is the solution of the exterior boundary value problem

$$
\begin{array}{cc}
\tau^{2} \Delta \mathbf{U}_{n}(\mathbf{r})+\left(1-\tau^{2}\right) \nabla\left(\nabla \cdot \mathbf{U}_{n}(\mathbf{r})\right)=\mathbf{0}, \quad \mathbf{r} \in V, \\
\mathbf{U}_{n}(\mathbf{r})=-\mathbf{P}_{n}(\mathbf{r}), \quad \mathbf{r} \in S, \\
\mathbf{U}_{n}(\mathbf{r})=O\left(\frac{1}{r}\right), \quad r \rightarrow+\infty
\end{array}
$$

Complete expansions for the scattering amplitudes are also obtained in [7] in the following form:

$$
\begin{aligned}
& g_{r}(\hat{\mathbf{r}}, \hat{\mathbf{k}})=\frac{1}{c_{p}^{2}} \hat{\mathbf{r}} \cdot \sum_{n=0}^{\infty} k^{n+1} \sum_{\rho=0}^{n} \mathbf{G}_{n, \rho}, \\
& g_{\theta}(\hat{\mathbf{r}}, \hat{\mathbf{k}})=\frac{1}{c_{s}^{2}} \hat{\boldsymbol{\jmath}} \cdot \sum_{n=0}^{\infty} k^{n+1} \sum_{\rho=0}^{n} \tau^{-(\rho+1)} \mathbf{G}_{n, \rho}, \\
& g_{\varphi}(\hat{\mathbf{r}}, \hat{\mathbf{k}})=\frac{1}{c_{s}^{2}} \hat{\boldsymbol{\varphi}} \cdot \sum_{n=0}^{\infty} k^{n+1} \sum_{\rho=0}^{n} \tau^{-(\rho+1)} \mathbf{G}_{n, \rho},
\end{aligned}
$$

where

$$
\mathbf{G}_{n, \rho}=\frac{1}{4 \pi} \frac{(i \tau)^{n+1}}{n !}\left(\begin{array}{l}
n \\
\rho
\end{array}\right)(-1)^{\rho+1} \int_{S} T_{\mathbf{r}^{\prime}} \boldsymbol{\Phi}_{n-\rho}\left(\mathbf{r}^{\prime}\right)\left(\hat{\mathbf{r}} \cdot \mathbf{r}^{\prime}\right)^{\rho} d s\left(\mathbf{r}^{\prime}\right) .
$$

Finally the low-frequency expansions for the scattering cross sections are obtained from (20) and (21) by substituting the expansions (32)-(34) for the amplitudes.

3. Papkovich-Grodski potentials in ellipsoidal geometry. In trying to evaluate the coefficients $\Phi_{0}, \Phi_{1}, \ldots$ of the low-frequency expansion (22) we only need to solve the boundary value problem (29)-(31) and find $\mathbf{U}_{n}$. From (24) we then obtain the coefficient $\boldsymbol{\Phi}_{n}$ by adding to $\mathbf{U}_{n}$ the function $\mathbf{P}_{n}$ which is expressed through the lower-order coefficients $\boldsymbol{\Phi}_{0}, \boldsymbol{\Phi}_{1}, \ldots, \boldsymbol{\Phi}_{n-1}$ as in (25). The coefficient $\boldsymbol{\Phi}_{n}$ forms the $n$ th-order field of $\boldsymbol{\Psi}$.

Equation (29) is the time-independent Navier equation in the absence of body forces. Therefore, by virtue of the Papkovich-Grodski [13] representation, there exist harmonic functions $\mathbf{A}^{n}$ and $B^{n}$, the vector and scalar Papkovich-Grodski potentials respectively, such that

$$
\begin{aligned}
\mathbf{U}_{n}(\mathbf{r})=\mathbf{A}^{n}(\mathbf{r})+\frac{\tau^{2}-1}{2} & \nabla\left(\mathbf{r} \cdot \mathbf{A}^{n}(\mathbf{r})+B^{n}(\mathbf{r})\right), \\
\Delta \mathbf{A}^{n}(\mathbf{r}) & =\mathbf{0}, \\
\Delta B^{n}(\mathbf{r}) & =0 .
\end{aligned}
$$

Since Eq. (29) is homogeneous the scalar potential $B^{n}$ is not necessary for the complete representation of $\mathbf{U}_{n}$. Nevertheless, by introducing $B^{n}$, which corresponds to a fictitious body force, it was made possible to reduce the calculations to a finite number of steps and actually describe how the low-frequency approximations can be evaluated in exact closed forms with the use of harmonic functions alone. This technique plays a crucial role in our 
work and it will be explored in detail after we introduce the ellipsoidal harmonic functions, i.e., the eigenfunctions that reflect the geometrical peculiarities of the scattering region. The ellipsoidal coordinates $(\rho, \mu, \nu)$ are related to the Cartesian coordinates $\left(x_{1}, x_{2}, x_{3}\right)$ by

$$
\begin{aligned}
& x_{1}=\frac{\rho \mu \nu}{h_{2} h_{3}}, \\
& x_{2}=\frac{\sqrt{\rho^{2}-h_{3}^{2}} \sqrt{\mu^{2}-h_{3}^{2}} \sqrt{h_{3}^{2}-\nu^{2}}}{h_{1} h_{3}}, \\
& x_{3}=\frac{\sqrt{\rho^{2}-h_{2}^{2}} \sqrt{h_{2}^{2}-\mu^{2}} \sqrt{h_{2}^{2}-\nu^{2}}}{h_{1} h_{2}},
\end{aligned}
$$

where

$$
h_{1}^{2}=\alpha_{2}^{2}-\alpha_{3}^{2}, \quad h_{2}^{2}=\alpha_{1}^{2}-\alpha_{3}^{2}, \quad h_{3}^{2}=\alpha_{1}^{2}-\alpha_{2}^{2},
$$

and

$$
0 \leqslant \nu^{2} \leqslant h_{3}^{2} \leqslant \mu^{2} \leqslant h_{2}^{2} \leqslant \rho^{2}<+\infty .
$$

For details we refer the reader to Hobson's book [10]. Separation of variables for the Laplace equation in ellipsoidal coordinates produces the interior ellipsoidal harmonics

$$
\mathbb{E}_{n}^{m}(\rho, \mu, \nu)=E_{n}^{m}(\rho) E_{n}^{m}(\mu) E_{n}^{m}(\nu)
$$

and the exterior ellipsoidal harmonics

$$
\mathbb{F}_{n}^{m}(\rho, \mu, \nu)=F_{n}^{m}(\rho) E_{n}^{m}(\mu) E_{n}^{m}(\nu),
$$

where $E_{n}^{m}$ are the Lamé functions of the first kind and

$$
F_{n}^{m}(\rho)=(2 n+1) E_{n}^{m}(\rho) I_{n}^{m}(\rho),
$$

with

$$
I_{n}^{m}(\rho)=\int_{\rho}^{+\infty} \frac{d u}{\left(E_{n}^{m}(u)\right)^{2} \sqrt{u^{2}-h_{2}^{2}} \sqrt{u^{2}-h_{3}^{2}}},
$$

are the Lamé functions of the second kind. The index $n$ specifies the degree of the corresponding ellipsoidal harmonic and takes the value $n=0,1,2,3, \ldots$ while $m$ represents the number of independent harmonic functions of degree $n$ and runs through the values $m=1,2, \ldots, 2 n+1$. The interior ellipsoidal harmonics of degree 0,1 , and 2 are those we use in the present work and for the sake of completeness we give their exact form, both in ellipsoidal as well as in Cartesian representation:

$$
\begin{aligned}
& \mathbb{E}_{0}^{1}(\rho, \mu, \nu)=1, \\
& \mathbb{E}_{1}^{1}(\rho, \mu, \nu)=\rho \mu \nu=x_{1} h_{2} h_{3}, \\
& \mathbb{E}_{1}^{2}(\rho, \mu, \nu)=\sqrt{\rho^{2}-h_{3}^{2}} \sqrt{\mu^{2}-h_{3}^{2}} \sqrt{h_{3}^{2}-\nu^{2}}=x_{2} h_{1} h_{3}, \\
& \mathbb{E}_{1}^{3}(\rho, \mu, \nu)=\sqrt{\rho^{2}-h_{2}^{2}} \sqrt{h_{2}^{2}-\mu^{2}} \sqrt{h_{2}^{2}-\nu^{2}}=x_{3} h_{1} h_{2},
\end{aligned}
$$




$$
\begin{aligned}
\mathbb{E}_{2}^{1}(\rho, \mu, \nu) & =\left(\rho^{2}-\alpha_{1}^{2}+\Lambda\right)\left(\mu^{2}-\alpha_{1}^{2}+\Lambda\right)\left(\nu^{2}-\alpha_{1}^{2}+\Lambda\right) \\
& =\left(\Lambda-\alpha_{1}^{2}\right)\left(\Lambda-\alpha_{2}^{2}\right)\left(\Lambda-\alpha_{3}^{2}\right)\left(\sum_{k=1}^{3} \frac{x_{k}^{2}}{\Lambda-\alpha_{k}^{2}}+1\right), \\
\mathbb{E}_{2}^{2}(\rho, \mu, \nu) & =\left(\rho^{2}-\alpha_{1}^{2}+\Lambda^{\prime}\right)\left(\mu^{2}-\alpha_{1}^{2}+\Lambda^{\prime}\right)\left(\nu^{2}-\alpha_{1}^{2}+\Lambda^{\prime}\right) \\
& =\left(\Lambda^{\prime}-\alpha_{1}^{2}\right)\left(\Lambda^{\prime}-\alpha_{2}^{2}\right)\left(\Lambda^{\prime}-\alpha_{3}^{2}\right)\left(\sum_{k=1}^{3} \frac{x_{k}^{2}}{\Lambda^{\prime}-\alpha_{k}^{2}}+1\right), \\
\mathbb{E}_{2}^{3}(\rho, \mu, \nu) & =\mathbb{E}_{1}^{1}(\rho, \mu, \nu) \mathbb{E}_{1}^{2}(\rho, \mu, \nu)=x_{1} x_{2} h_{1} h_{2} h_{3}^{2}, \\
\mathbb{E}_{2}^{4}(\rho, \mu, \nu) & =\mathbb{E}_{1}^{1}(\rho, \mu, \nu) \mathbb{E}_{1}^{3}(\rho, \mu, \nu)=x_{1} x_{3} h_{1} h_{2}^{2} h_{3}, \\
\mathbb{E}_{2}^{5}(\rho, \mu, \nu) & =\mathbb{E}_{1}^{2}(\rho, \mu, \nu) \mathbb{E}_{1}^{3}(\rho, \mu, \nu)=x_{2} x_{3} h_{1}^{2} h_{2} h_{3},
\end{aligned}
$$

where $\Lambda, \Lambda^{\prime}$ are the two roots of the equation

$$
\sum_{i=1}^{3} \frac{1}{\Lambda-\alpha_{i}^{2}}=0
$$

The exterior ellipsoidal harmonics of degree 0,1 , and 2 are given from (42) when (45)-(47) are used. The Lamé functions of degree 0, 1, and 2 that appear in the expression (44) for the elliptic integrals $I_{n}^{m}(\rho)$ are

$$
\begin{aligned}
& E_{0}^{1}(\rho)=1, \\
& E_{1}^{m}(\rho)=\sqrt{\rho^{2}-\alpha_{1}^{2}+\alpha_{m}^{2}}, \quad m=1,2,3, \\
& E_{2}^{1}(\rho)=\rho^{2}-\alpha_{1}^{2}+\Lambda, \\
& E_{2}^{2}(\rho)=\rho^{2}-\alpha_{1}^{2}+\Lambda^{\prime}, \\
& E_{2}^{3}(\rho)=\rho \sqrt{\rho^{2}-h_{3}^{2}}, \\
& E_{2}^{4}(\rho)=\rho \sqrt{\rho^{2}-h_{2}^{2}}, \\
& E_{2}^{5}(\rho)=\sqrt{\rho^{2}-h_{2}^{2}} \sqrt{\rho^{2}-h_{3}^{2}} .
\end{aligned}
$$

For detailed analysis of the ellipsoidal harmonic functions and the relative useful properties see [2].

The set of functions

$$
\left\{E_{n}^{m}(\mu) E_{n}^{m}(\nu): n=0,1,2, \ldots, m=1,2,3, \ldots, 2 n+1\right\}
$$

forms a complete orthogonal set of surface harmonics on the surface of the ellipsoid

$$
\frac{x_{1}^{2}}{\rho^{2}}+\frac{x_{2}^{2}}{\rho^{2}-h_{3}^{2}}+\frac{x_{3}^{2}}{\rho^{2}-h_{2}^{2}}=1,
$$

which coincides with the surface of the scatterer (1) whenever $\rho=\alpha_{1}$.

The vector and scalar Papkovich-Grodski potentials $\mathbf{A}^{n}$ and $B^{n}$, in the representation (36), assume the following expansions in terms of surface ellipsoidal harmonics

$$
\mathbf{A}^{\prime}=\sum_{n=0}^{\infty} \sum_{m=1}^{2 n+1} \mathbf{c}_{n}^{l, m} \mathbb{F}_{n}^{m}(\rho, \mu, \nu)
$$




$$
B^{\prime}=\sum_{n=0}^{\infty} \sum_{m=1}^{2 n+1} \beta_{n}^{l, m} \mathbb{F}_{n}^{m}(\rho, \mu, \nu),
$$

which satisfy via (36) the asymptotic relation (31).

Since

$$
\nabla\left(\mathbf{r} \cdot \mathbf{A}^{\prime}\right)=\mathbf{A}^{l}+\sum_{n=0}^{\infty} \sum_{m=1}^{2 n+1}\left(\mathbf{r} \cdot \mathbf{c}_{n}^{l, m}\right) \nabla \mathbb{F}_{n}^{m}(\rho, \mu, \nu),
$$

the representation (36), in view of the expansions (51), (52), provides

$$
\begin{aligned}
\mathbf{U}_{l}(\mathbf{r})= & \frac{1}{2}\left(\tau^{2}+1\right) \sum_{n=0}^{\infty} \sum_{m=1}^{2 n+1} \mathbf{c}_{n}^{l, m} \mathbb{F}_{n}^{m}(\rho, \mu, \nu) \\
& +\frac{1}{2}\left(\tau^{2}-1\right) \sum_{n=0}^{\infty} \sum_{m=1}^{2 n+1}\left(\mathbf{r} \cdot \mathbf{c}_{n}^{l, m}+\beta_{n}^{l, m}\right) \nabla \mathbb{F}_{n}^{m}(\rho, \mu, \nu) .
\end{aligned}
$$

The expansion (54) has to become equal to $-\mathbf{P}_{/}(\mathbf{r})$ on the surface $S$ of the scatterer. From (25) and (27) we observe that $\mathbf{P}_{/}(\mathbf{r})$ is of degree $l$; therefore, $\mathbf{U}_{l}(\mathbf{r})$ has to be of degree $l$ in the surface variables $\mu, \nu$. This implies that all the coefficients $\mathbf{c}_{n}^{l, m}$ for $n>l$ and $\beta_{n}^{l, m}$ for $n>l+1$, vanish, and hence the expansion (54) degenerates to a finite sum.

If we apply the gradient operator on the exterior ellipsoidal harmonics we obtain

$$
\begin{aligned}
\nabla \mathbb{F}_{n}^{m}(\rho, \mu, \nu)= & (2 n+1)\left(\nabla \mathbb{E}_{n}^{m}(\rho, \mu, \nu)\right) I_{n}^{m}(\rho) \\
& -(2 n+1) \frac{\hat{\rho}}{h_{\rho}} \frac{\mathbb{E}_{n}^{m}(\rho, \mu, \nu)}{\left[E_{n}^{m}(\rho)\right]^{2} \sqrt{\rho^{2}-h_{2}^{2}} \sqrt{\rho^{2}-h_{3}^{2}}},
\end{aligned}
$$

where

$$
h_{\rho}=\frac{\sqrt{\rho^{2}-\mu^{2}} \sqrt{\rho^{2}-\nu^{2}}}{\sqrt{\rho^{2}-h_{2}^{2}} \sqrt{\rho^{2}-h_{3}^{2}}}
$$

is the square root of the ellipsoidal metric coefficient that corresponds to the variable $\rho$,

$$
\hat{\boldsymbol{\rho}}=\frac{\rho}{h_{\rho}} \sum_{i=1}^{3} \frac{x_{i}}{\rho^{2}-\alpha_{1}^{2}+\alpha_{i}^{2}} \hat{\mathbf{x}}_{i}
$$

is the unit curvilinear vector relative to the variable $\rho$, and $\hat{\mathbf{x}}_{i}, i=1,2,3$, are the Cartesian base vectors.

Inserting (55) into the finite nonvanishing part of the expansion (54) we conclude

$$
\begin{aligned}
\mathbf{U}_{l}(\mathbf{r})= & \frac{1}{2}\left\{\left(\tau^{2}+1\right) \sum_{n=0}^{l} \sum_{m=1}^{2 n+1} \mathbf{c}_{n}^{l, m}(2 n+1) I_{n}^{m}(\rho) \mathbb{E}_{n}^{m}(\rho, \mu, \nu)\right. \\
& +\left(\tau^{2}-1\right) \sum_{n=0}^{l} \sum_{m=1}^{2 n+1}\left(\mathbf{r} \cdot \mathbf{c}_{n}^{l, m}\right)(2 n+1) I_{n}^{m}(\rho) \nabla \mathbb{E}_{n}^{m}(\rho, \mu, \nu) \\
& \left.+\left(\tau^{2}-1\right) \sum_{n=0}^{l+1} \sum_{m=1}^{2 n+1} \beta_{n}^{l, m}(2 n+1) I_{n}^{m}(\rho) \nabla \mathbb{E}_{n}^{m}(\rho, \mu, \nu)\right\}
\end{aligned}
$$


(Continued)

$$
\begin{aligned}
-\frac{1}{2}\left(\tau^{2}-1\right) \frac{\hat{\boldsymbol{\rho}}}{\sqrt{\rho^{2}-\mu^{2}} \sqrt{\rho^{2}-\nu^{2}}} & \left\{\sum_{n=0}^{l} \sum_{m=1}^{2 n+1}\left(\mathbf{r} \cdot \mathbf{c}_{n}^{l, m}\right)(2 n+1)\left(E_{n}^{m}(\rho)\right)^{-2} \mathbb{E}_{n}^{m}(\rho, \mu, \nu)\right. \\
+ & \left.\sum_{\eta=0}^{l+1} \sum_{m=1}^{2 n+1} \beta_{n}^{l, m}(2 n+1)\left(E_{n}^{m}(\rho)\right)^{-2} \mathbb{E}_{n}^{m}(\rho, \mu, \nu)\right\} .
\end{aligned}
$$

The three sums in the first braces in the right-hand side of (58) are of degree $l$ in the variables $\mu, \nu$ of the surface harmonics $E_{n}^{m}(\mu) E_{n}^{m}(\nu)$ [10]. The two sums inside the second braces are of degree $l+1$.

We observe that due to the factor $\hat{\rho}\left(\rho^{2}-\mu^{2}\right)^{-1 / 2}\left(\rho^{2}-\nu^{2}\right)^{-1 / 2}$ it is not possible to express the second braces in the right-hand side of (58) in terms of a finite expression of surface ellipsoidal harmonics. Therefore, in practice, it is not possible to evaluate the coefficients explicitly and obtain the solution in closed form.

Nevertheless, since $\mathbf{A}^{\prime}$ and $B^{\prime}$ are not independent [13], the corresponding coefficients $\mathbf{c}_{n}^{l, m}$ and $\beta_{n}^{l, m}$ are also not independent and we can choose to express the $\beta$ 's in terms of the c's in such a way that the second sum in the right-hand side of (58) vanishes for $\mathbf{r} \in S$. When this is done the coefficients $\mathbf{c}_{n}^{l, m}$ (and through these the coefficients $\beta_{n}^{l, m}$ ) can be evaluated from the boundary condition (30). Therefore, along our line of work, the introduction of the fictitious body force that is represented by the scalar potential $B^{\prime}$ is indispensable and forms the key to our method.

In following this program a considerable work has to be done in order to express the

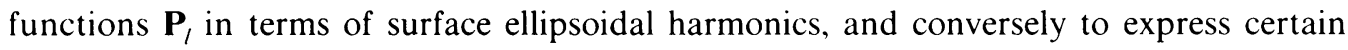
combinations of ellipsoidal harmonics in appropriate Cartesian form.

4. The zeroth-order field. The zeroth-order field is obtained as the solution of the exterior boundary value problem

$$
\begin{array}{ll}
\boldsymbol{\Phi}_{0}(\mathbf{r})=\pi^{0}+\mathbf{U}_{0}(\mathbf{r}), & \rho>\alpha_{1}, \\
\boldsymbol{\Phi}_{0}(\mathbf{r})=\mathbf{0}, \quad \rho=\alpha_{1}, & \\
\boldsymbol{\Phi}_{0}(\mathbf{r})=\pi^{0}+O\left(\frac{1}{\rho}\right), & \rho \rightarrow+\infty,
\end{array}
$$

where, as we proved in Paragraph $3, \mathbf{U}_{0}(\mathbf{r})$ has the following complete representation in terms of surface ellipsoidal harmonics:

$$
\begin{aligned}
\mathbf{U}_{0}(\rho, \mu, \nu)= & \frac{1}{2}\left(\tau^{2}-1\right)\left\{\frac{\tau^{2}+1}{\tau^{2}-1} \mathbf{c}_{0}^{0.1} I_{0}^{1}(\rho)+3 h_{1} h_{2} h_{3} \sum_{m=1}^{3} \frac{\beta_{1}^{0, m}}{h_{m}} I_{1}^{m}(\rho) \hat{\mathbf{x}}_{m}\right\} \\
& -\frac{1}{2}\left(\tau^{2}-1\right) \mathbf{R}^{e}\left\{\beta_{0}^{0.1}+\sum_{m=1}^{3}\left(\frac{c_{0 m}^{0.1} h_{m} E_{1}^{m}(\rho)}{h_{1} h_{2} h_{3}}+\frac{3 \beta_{1}^{0 . m}}{E_{1}^{m}(\rho)}\right) E_{1}^{m}(\mu) E_{1}^{m}(\nu)\right\}
\end{aligned}
$$

and

$$
\mathbf{R}^{e}=\left(\rho^{2}-\mu^{2}\right)^{-1 / 2}\left(\rho^{2}-\nu^{2}\right)^{-1 / 2} \hat{\rho} .
$$


We will refer to the first braces of (62) as the "Cartesian part" of $\mathbf{U}_{0}$ and to the second braces as the "ellipsoidal part" of $\mathbf{U}_{0}$.

For $\rho=\alpha_{1}$, the vanishing of the ellipsoidal part of $\mathbf{U}_{0}$ and the orthogonality of the surface ellipsoidal harmonics imply

$$
\left.\begin{array}{l}
\beta_{0}^{0.1}=0, \\
\beta_{1}^{0, m}=-\frac{h_{m} \alpha_{m}^{2}}{3 h_{1} h_{2} h_{3}} c_{0 m}^{0.1} . \quad m=1,2,3 .
\end{array}\right\}
$$

Then the field $\boldsymbol{\Phi}_{0}$ takes the form

$$
\begin{aligned}
\boldsymbol{\Phi}_{0}= & \pi^{0}-\frac{1}{2} \sum_{m=1}^{3}\left[\left(\tau^{2}-1\right) \alpha_{m}^{2} I_{1}^{m}(\rho)-\left(\tau^{2}+1\right) I_{0}^{1}(\rho)\right] c_{0 m}^{0.1} \hat{\mathbf{x}}_{m} \\
& -\frac{\tau^{2}-1}{2} \frac{\mathbf{R}^{e}}{h_{1} h_{2} h_{3}} \sum_{m=1}^{3}\left[E_{1}^{m}(\rho)-\frac{\alpha_{m}^{2}}{E_{1}^{m}(\rho)}\right] h_{m} c_{0 m}^{0.1} E_{1}^{m}(\mu) E_{1}^{m}(\nu)
\end{aligned}
$$

and (60) implies that

$$
c_{0 m}^{0.1}=\frac{2 \pi_{m}^{0}}{L_{0}^{m}}, \quad m=1,2,3,
$$

where

$$
L_{0}^{m}(\rho)=\left(\tau^{2}-1\right) \alpha_{m}^{2} I_{1}^{m}(\rho)-\left(\tau^{2}+1\right) I_{0}^{1}(\rho), \quad m=1,2,3,
$$

and $L_{0}^{\prime \prime}$ denotes the value of the function $L_{0}^{m}(\rho)$ on the surface of the scatterer $\left(\rho=\alpha_{1}\right)$.

Similarly $I_{n}^{m}=I_{n}^{m}\left(\alpha_{1}\right)$.

Inserting the values (66) for the coefficients $c_{0 m}^{0.1}$ into (65) we finally obtain the zeroth-order field in the following form, which explicitly indicates the dependence of $\boldsymbol{\Phi}_{0}$ on the polarization vector $\pi^{0}$ and the direction of observation $\mathbf{r}$ :

$$
\begin{aligned}
\boldsymbol{\Phi}_{0}(\mathbf{r})= & \pi^{0} \cdot \sum_{m=1}^{3}\left[1-\frac{L_{0}^{m}(\rho)}{L_{0}^{m}}\right] \hat{\mathbf{x}}_{m} \otimes \hat{\mathbf{x}}_{m} \\
& -\left(\tau^{2}-1\right)\left(\rho^{2}-\alpha_{1}^{2}\right) \mathbf{R}^{e} \otimes \pi^{0} \cdot \sum_{m=1}^{3} \frac{\hat{\mathbf{x}}_{m} \otimes \hat{\mathbf{x}}_{m}}{\left(\rho^{2}-\alpha_{1}^{2}+\alpha_{m}^{2}\right) L_{0}^{m}} \cdot \mathbf{r} .
\end{aligned}
$$

For the case of longitudinal incidence $\pi^{0}=\hat{\mathbf{k}}$, while for transverse incidence $\pi^{0}=\hat{\mathbf{b}}$.

5. The first-order field. The exterior boundary value problem that determines the first-order field $\boldsymbol{\Phi}_{1}$ is the following:

$$
\begin{array}{ll}
\boldsymbol{\Phi}_{1}(\mathbf{r})=\mathbf{P}_{1}(\mathbf{r})+\mathbf{U}_{1}(\mathbf{r}), & \rho>\alpha_{1}, \\
\boldsymbol{\Phi}_{1}(\mathbf{r})=\mathbf{0}, \quad \rho=\alpha_{1}, & \\
\boldsymbol{\Phi}_{1}(\mathbf{r})=\mathbf{P}_{1}(\mathbf{r})+O\left(\frac{1}{\rho}\right), & \rho \rightarrow+\infty,
\end{array}
$$

where

$$
\mathbf{P}_{1}(\mathbf{r})=\pi^{1} \otimes \hat{\mathbf{k}} \cdot \mathbf{r}-\frac{\tau^{3}+2}{12 \pi \bar{\mu} \tau} \int_{\rho=\alpha_{1}} T \Phi_{0}(\mathbf{r}) d s(\mathbf{r}) .
$$

The representation of $\mathbf{U}_{1}(\mathbf{r})$ in terms of surface ellipsoidal harmonics can be obtained 
from Paragraph 3 in the following form

where

$$
\mathbf{U}_{1}(\mathbf{r})=\mathbf{U}_{1}^{\text {cart }}(\mathbf{r})+\mathbf{U}_{1}^{\text {ellip }}(\mathbf{r}),
$$

$$
\begin{aligned}
\mathbf{U}_{1}^{\text {cart }}=\frac{\tau^{2}-1}{2}\left\{\left[\frac{\tau^{2}+1}{\tau^{2}-1} \mathbf{c}_{0}^{1.1} I_{0}^{1}(\rho)+3 h_{1} h_{2} h_{3} \sum_{k=1}^{3} \beta_{1}^{1 . k} I_{1}^{k}(\rho) \frac{\hat{\mathbf{x}}_{k}}{h_{k}}\right]\right. \\
+\sum_{n=1}^{3}\left[3 \frac{\tau^{2}+1}{\tau^{2}-1} \mathbf{c}_{1}^{1 . n} I_{1}^{n}(\rho)+3 \sum_{k=1}^{3} \frac{h_{n}}{h_{k}} c_{1 n}^{1 . k} I_{1}^{k}(\rho) \hat{\mathbf{x}}_{k}\right. \\
+10 \beta_{2}^{1.1} I_{2}^{1}(\rho) \frac{h_{n}}{h_{1} h_{2} h_{3}} \frac{\left(\Lambda-\alpha_{1}^{2}\right)\left(\Lambda-\alpha_{2}^{2}\right)\left(\Lambda-\alpha_{3}^{2}\right)}{\Lambda-\alpha_{n}^{2}} \hat{\mathbf{x}}_{n} \\
\left.+10 \beta_{2}^{1.2} I_{2}^{2}(\rho) \frac{h_{n}}{h_{1} h_{2} h_{3}} \frac{\left(\Lambda^{\prime}-\alpha_{1}^{2}\right)\left(\Lambda^{\prime}-\alpha_{2}^{2}\right)\left(\Lambda^{\prime}-\alpha_{3}^{2}\right)}{\Lambda^{\prime}-\alpha_{n}^{2}} \hat{\mathbf{x}}_{n}\right] \mathbb{E}_{1}^{n}(\rho, \mu, \nu) \\
+5 h_{1} h_{2} h_{3}\left(\beta_{2}^{1.3} I_{2}^{3}(\rho) \frac{\hat{\mathbf{x}}_{2}}{h_{2}}+\beta_{2}^{1.4} I_{2}^{4}(\rho) \frac{\hat{\mathbf{x}}_{3}}{h_{3}}\right) \mathbb{E}_{1}^{1}(\rho, \mu, \nu) \\
+5 h_{1} h_{2} h_{3}\left(\beta_{2}^{1.3} I_{2}^{3}(\rho) \frac{\hat{\mathbf{x}}_{1}}{h_{1}}+\beta_{2}^{1.5} I_{2}^{5}(\rho) \frac{\hat{\mathbf{x}}_{3}}{h_{3}}\right) \mathbb{E}_{1}^{2}(\rho, \mu, \nu) \\
\left.+5 h_{1} h_{2} h_{3}\left(\beta_{2}^{1.4} I_{2}^{4}(\rho) \frac{\hat{\mathbf{x}}_{1}}{h_{1}}+\beta_{2}^{1.5} I_{2}^{5}(\rho) \frac{\hat{\mathbf{x}}_{2}}{h_{2}}\right) \mathbb{E}_{1}^{3}(\rho, \mu, \nu)\right\}, \quad(74)
\end{aligned}
$$$$
\mathbf{U}_{1}^{\text {cllip }}(\mathbf{r})=-\frac{\tau^{2}-1}{2} \mathbf{R}^{e}\left\{\left[h_{1} h_{2} h_{3} \sum_{k=1}^{3} \frac{1}{h_{k}} c_{1 k}^{1, k}+\beta_{0}^{1.1}\right]\right.
$$$$
+\sum_{n=1}^{3}\left[\frac{h_{n}}{h_{1} h_{2} h_{3}} c_{0 n}^{1,1}+3 \beta_{1}^{1, n} \frac{1}{\left(E_{1}^{n}(\rho)\right)^{2}}\right] \mathbb{E}_{1}^{n}(\rho, \mu, \nu)
$$$$
+\left[\frac{5 \beta_{2}^{1,1}}{E_{2}^{1}(\rho)}-\frac{3}{h_{1} h_{2} h_{3}\left(\Lambda-\Lambda^{\prime}\right)}\left(c_{11}^{1,1} h_{1}\left(\Lambda^{\prime}-\alpha_{1}^{2}\right)\right.\right.
$$$$
\left.\left.-c_{12}^{1.2} h_{2}\left(\Lambda^{\prime}-\alpha_{2}^{2}\right)+c_{13}^{1.3} h_{3}\left(\Lambda^{\prime}-\alpha_{3}^{2}\right)\right)\right] E_{2}^{1}(\mu) E_{2}^{1}(\nu)
$$$$
+\left[\frac{5 \beta_{2}^{1.2}}{E_{2}^{2}(\rho)}+\frac{3}{h_{1} h_{2} h_{3}\left(\Lambda-\Lambda^{\prime}\right)}\left(c_{11}^{1,1} h_{1}\left(\Lambda-\alpha_{1}^{2}\right)\right.\right.
$$$$
\left.\left.-c_{12}^{1,2} h_{2}\left(\Lambda-\alpha_{2}^{2}\right)+c_{13}^{1.3} h_{3}\left(\Lambda-\alpha_{3}^{2}\right)\right)\right] E_{2}^{2}(\mu) E_{2}^{2}(\nu)
$$$$
+\left[\frac{5 \beta_{2}^{1,3}}{\left(E_{2}^{3}(\rho)\right)^{2}}+\frac{3}{h_{3}}\left(\frac{c_{12}^{1,1}}{h_{1}\left(E_{1}^{1}(\rho)\right)^{2}}+\frac{c_{11}^{1.2}}{h_{2}\left(E_{1}^{2}(\rho)\right)^{2}}\right)\right] \mathbb{E}_{2}^{3}(\rho, \mu, \nu)
$$$$
+\left[\frac{5 \beta_{2}^{1.4}}{\left(E_{2}^{4}(\rho)\right)^{2}}+\frac{3}{h_{2}}\left(\frac{c_{13}^{1.1}}{h_{1}\left(E_{1}^{1}(\rho)\right)^{2}}+\frac{c_{11}^{1.3}}{h_{3}\left(E_{1}^{3}(\rho)\right)^{2}}\right)\right] \mathbb{E}_{2}^{4}(\rho, \mu, \nu)
$$$$
\left.+\left[\frac{5 \beta_{2}^{1.5}}{\left(E_{2}^{5}(\rho)\right)^{2}}+\frac{3}{h_{1}}\left(\frac{c_{12}^{1.3}}{h_{3}\left(E_{1}^{3}(\rho)\right)^{2}}+\frac{c_{13}^{1.2}}{h_{2}\left(E_{1}^{2}(\rho)\right)^{2}}\right)\right] \mathbb{E}_{2}^{5}(\rho, \mu, \nu)\right\} \text {. }
$$ 
In order to be able to apply the boundary condition (70) we need to express the function $\mathbf{P}_{1}(\mathbf{r})$ in terms of surface ellipsoidal harmonics.

We first evaluate the surface integral in (72). Using $\Phi_{0}$ in the form

$$
\begin{aligned}
\boldsymbol{\Phi}_{0}(r)= & \pi^{0}-\frac{1}{2} \sum_{m=1}^{3} L_{0}^{m}(\rho) c_{0 m}^{0,1} \hat{\mathbf{x}}_{m} \\
& +\frac{1}{2}\left(\tau^{2}-1\right) \frac{\hat{\boldsymbol{\rho}}}{h_{\rho}} \sum_{m=1}^{3} \frac{\partial}{\partial \rho}\left(I_{0}^{1}(\rho)-\alpha_{m}^{2} I_{1}^{m}(\rho)\right) x_{m} c_{0 m}^{0.1},
\end{aligned}
$$

we obtain, after long calculations,

$$
\begin{aligned}
\left.T \boldsymbol{\Phi}_{0}\right|_{\rho=\alpha_{1}}= & \left.2 \bar{\mu} \hat{\boldsymbol{\rho}} \cdot \nabla \boldsymbol{\Phi}_{0}\right|_{p=\alpha_{1}}+\left.\lambda \hat{\boldsymbol{\rho}} \nabla \cdot \boldsymbol{\Phi}_{0}\right|_{\rho=\alpha_{1}} \\
& +\bar{\mu} \hat{\boldsymbol{\rho}} \times\left.\left(\nabla \times \boldsymbol{\Phi}_{0}\right)\right|_{\rho=\alpha_{1}} \\
= & \left.2 \bar{\mu}\left(\alpha_{1}^{2}-\mu^{2}\right)^{-1 / 2}\left(\alpha_{1}^{2}-\nu^{2}\right)^{-1 / 2}\left[-\mathbf{c}_{0}^{0,1}+\frac{\lambda+\bar{\mu}}{\lambda+2 \bar{\mu}} \hat{\boldsymbol{\rho}} \otimes \hat{\boldsymbol{\rho}} \cdot \mathbf{c}_{0}^{0.1}\right]\right|_{\rho=\alpha_{1}} \\
& -\left.\frac{\lambda \bar{\mu}}{\lambda+2 \bar{\mu}}\left(\alpha_{1}^{2}-\mu^{2}\right)^{-1 / 2}\left(\alpha_{1}^{2}-\nu^{2}\right)^{-1 / 2} \hat{\boldsymbol{\rho}} \otimes \hat{\boldsymbol{\rho}} \cdot \mathbf{c}_{0}^{0.1}\right|_{\rho=\alpha_{1}} \\
& +\left.\bar{\mu}\left(\alpha_{1}^{2}-\mu^{2}\right)^{-1 / 2}\left(\alpha_{1}^{2}-\nu^{2}\right)^{-1 / 2}\left[\mathbf{c}_{0}^{0.1}-\hat{\boldsymbol{\rho}} \otimes \hat{\boldsymbol{\rho}} \cdot \mathbf{c}_{0}^{0.1}\right]\right|_{\rho=\alpha_{1}} \\
= & -\bar{\mu}\left(\alpha_{1}^{2}-\mu^{2}\right)^{-1 / 2}\left(\alpha_{1}^{2}-\nu^{2}\right)^{-1 / 2} \mathbf{c}_{0}^{0.1} .
\end{aligned}
$$

In [2] we can find the value of the integral

$$
\int_{\rho=\alpha_{1}}\left(\rho^{2}-\mu^{2}\right)^{-1 / 2}\left(\rho^{2}-\nu^{2}\right)^{-1 / 2} d s=4 \pi
$$

and from (46), (72), (77), and (78) we conclude that

$$
\mathbf{P}_{1}(\mathbf{r})=\frac{\pi^{1}}{h_{1} h_{2} h_{3}} \sum_{n=1}^{3} i_{n} h_{n} \mathbb{E}_{1}^{n}(\rho, \mu, \nu)+\frac{2\left(\tau^{3}+2\right)}{3 \tau} \sum_{n=1}^{3} \frac{\pi_{n}^{0}}{L_{0}^{n}} \hat{\mathbf{x}}_{n} .
$$

The vanishing of the ellipsoidal part $\mathbf{U}_{1}^{\text {ellip }}\left(\rho=\alpha_{1}\right)$ as it is given by (75) provides the following relations between the $\beta$ 's and the c's coefficients:

$$
\begin{aligned}
\beta_{0}^{1.1}= & -h_{1} h_{2} h_{3} \sum_{k=1}^{3} \frac{1}{h_{k}} c_{1 k}^{1, k}, \\
\beta_{1}^{1, k}= & -\frac{h_{k} \alpha_{k}^{2}}{3 h_{1} h_{2} h_{3}} c_{0 k}^{1.1}, \quad k=1,2,3, \\
\beta_{2}^{1.1}= & \frac{3 \Lambda}{5 h_{1} h_{2} h_{3}\left(\Lambda-\Lambda^{\prime}\right)} \\
& \cdot\left[c_{11}^{1.1} h_{1}\left(\Lambda^{\prime}-\alpha_{1}^{2}\right)-c_{12}^{1.2} h_{2}\left(\Lambda^{\prime}-\alpha_{2}^{2}\right)+c_{13}^{1.3} h_{3}\left(\Lambda^{\prime}-\alpha_{3}^{2}\right)\right], \\
\beta_{2}^{1.2}= & \frac{-3 \Lambda^{\prime}}{5 h_{1} h_{2} h_{3}\left(\Lambda-\Lambda^{\prime}\right)} \\
& \cdot\left[c_{11}^{1,1} h_{1}\left(\Lambda-\alpha_{1}^{2}\right)-c_{12}^{1.2} h_{2}\left(\Lambda-\alpha_{2}^{2}\right)+c_{13}^{1.3} h_{3}\left(\Lambda-\alpha_{3}^{2}\right)\right],
\end{aligned}
$$




$$
\begin{aligned}
& \beta_{2}^{1,3}=-\frac{3}{5 h_{3}}\left(\frac{\alpha_{1}^{2}}{h_{2}} c_{11}^{1,2}+\frac{\alpha_{2}^{2}}{h_{1}} c_{12}^{1,1}\right), \\
& \beta_{2}^{1.4}=-\frac{3}{5 h_{2}}\left(\frac{\alpha_{1}^{2}}{h_{3}} c_{11}^{1.3}+\frac{\alpha_{3}^{2}}{h_{1}} c_{13}^{1,1}\right), \\
& \beta_{2}^{1.5}=-\frac{3}{5 h_{1}}\left(\frac{\alpha_{2}^{2}}{h_{3}} c_{12}^{1.3}+\frac{\alpha_{3}^{2}}{h_{2}} c_{13}^{1,2}\right) .
\end{aligned}
$$

Long and tedious calculations on the expression

$$
\mathbf{P}_{1}\left(\rho=\alpha_{1}\right)+\mathbf{U}_{1}^{\text {cart }}\left(\rho=\alpha_{1}\right)=\mathbf{0},
$$

by means of the orthogonality of the surface ellipsoidal harmonics, yield the following values of the coefficients:

$$
\begin{aligned}
& c_{0 n}^{1,1}=\frac{4\left(\tau^{3}+2\right)}{3 \tau\left(L_{0}^{n}\right)^{2}} \pi_{n}^{0}, \quad n=1,2,3, \\
& c_{1 n}^{1, n}=\frac{h_{n}}{3 h_{1} h_{2} h_{3}} \pi^{1} \otimes \hat{\mathbf{k}}: \tilde{\Gamma}_{n}, \quad n=1,2,3, \\
& c_{1 k}^{1, n}=-\frac{h_{n}}{3 h_{1} h_{2} h_{3} \Delta_{n k}} \pi^{1} \otimes \hat{\mathbf{k}}: \tilde{N}_{n k}, \quad n, k=1,2,3, n \neq k,
\end{aligned}
$$

where the double contraction is defined by

$$
\begin{aligned}
& (\boldsymbol{\alpha} \otimes \mathbf{b}):(\mathbf{c} \otimes \mathbf{d})=(\mathbf{b} \cdot \mathbf{c})(\boldsymbol{\alpha} \cdot \mathbf{d}), \\
& \tilde{\Gamma}_{n}=\frac{\left(\tau^{2}-1\right)^{2}}{\Delta} \Lambda \Lambda^{\prime} I_{2}^{1} I_{2}^{2} \tilde{\mathrm{I}}+\frac{3 \tau^{4}}{\Delta} \frac{I_{1}^{1} I_{1}^{2} I_{1}^{3}}{I_{1}^{n}} \hat{\mathbf{x}}_{n} \otimes \hat{\mathbf{x}}_{n} \\
& +\frac{\tau^{2}\left(\tau^{2}-1\right)}{\Delta} \tilde{Z}_{n}, \quad n=1,2,3 \text {, } \\
& \Delta=-\tau^{2}\left(\tau^{2}-1\right)^{2} \Lambda \Lambda^{\prime} \frac{I_{2}^{1} I_{2}^{2}}{\alpha_{1} \alpha_{2} \alpha_{3}}-3 \tau^{6} I_{1}^{1} I_{1}^{2} I_{1}^{3} \\
& -\tau^{4}\left(\tau^{2}-1\right) I_{1}^{1} I_{1}^{2} I_{1}^{3} \sum_{k=1}^{3} \frac{M_{k k}}{I_{1}^{k}}, \\
& M_{n k}(\rho)=\frac{1}{\Lambda-\Lambda^{\prime}}\left[\frac{\Lambda\left(\Lambda-\alpha_{1}^{2}\right)\left(\Lambda-\alpha_{2}^{2}\right)\left(\Lambda-\alpha_{3}^{2}\right)}{\left(\Lambda-\alpha_{n}^{2}\right)\left(\Lambda-\alpha_{k}^{2}\right)} I_{2}^{1}(\rho)\right. \\
& \left.-\frac{\Lambda^{\prime}\left(\Lambda^{\prime}-\alpha_{1}^{2}\right)\left(\Lambda^{\prime}-\alpha_{2}^{2}\right)\left(\Lambda^{\prime}-\alpha_{3}^{2}\right)}{\left(\Lambda^{\prime}-\alpha_{n}^{2}\right)\left(\Lambda^{\prime}-\alpha_{k}^{2}\right)} I_{2}^{2}(\rho)\right], \\
& M_{n k}=M_{n k}\left(\alpha_{1}\right), \quad n, k=1,2,3, \\
& \tilde{Z}_{1}=\left(I_{1}^{2} M_{33}+I_{1}^{3} M_{22}\right) \hat{\mathbf{x}}_{1} \otimes \hat{\mathbf{x}}_{1}-I_{1}^{3} M_{12} \hat{\mathbf{x}}_{2} \otimes \hat{\mathbf{x}}_{2}-I_{1}^{2} M_{31} \hat{\mathbf{x}}_{3} \otimes \hat{\mathbf{x}}_{3}, \\
& \tilde{Z}_{2}=-I_{1}^{3} M_{12} \hat{\mathbf{x}}_{1} \otimes \hat{\mathbf{x}}_{1}+\left(I_{1}^{1} M_{33}+I_{1}^{3} M_{11}\right) \hat{\mathbf{x}}_{2} \otimes \hat{\mathbf{x}}_{2}-I_{1}^{1} M_{32} \hat{\mathbf{x}}_{3} \otimes \hat{\mathbf{x}}_{3} \text {, } \\
& \tilde{Z}_{3}=-I_{1}^{2} M_{31} \hat{\mathbf{x}}_{1} \otimes \hat{\mathbf{x}}_{1}-I_{1}^{1} M_{32} \hat{\mathbf{x}}_{2} \otimes \hat{\mathbf{x}}_{2}+\left(I_{1}^{1} M_{22}+I_{1}^{2} M_{11}\right) \hat{\mathbf{x}}_{3} \otimes \hat{\mathbf{x}}_{3} \text {, }
\end{aligned}
$$




$$
\begin{aligned}
& \tilde{N}_{n k}=\left(\tau^{2}-1\right)\left(\hat{\mathbf{x}}_{n} \otimes \hat{\mathbf{x}}_{k}-\hat{\mathbf{x}}_{k} \otimes \hat{\mathbf{x}}_{n}\right) L_{1}^{n k}+2 \hat{\mathbf{x}}_{n} \otimes \hat{\mathbf{x}}_{k} I_{1}^{k}, \\
& n, k=1,2,3, k \neq n \text {, } \\
& L_{1}^{k n}(\rho)=\frac{\alpha_{k}^{2} I_{1}^{k}(\rho)-\alpha_{n}^{2} I_{1}^{n}(\rho)}{\alpha_{k}^{2}-\alpha_{n}^{2}} \\
& L_{1}^{k n}=L_{1}^{k n}\left(\alpha_{1}\right), \quad k, n=1,2,3, k \neq n, \\
& \left.\begin{array}{r}
\Delta_{n k}=\left(\tau^{2}+1\right) I_{1}^{n} I_{1}^{k}+\left(\tau^{2}-1\right) \frac{\alpha_{n}^{2} I_{1}^{n} I_{1}^{n}-\alpha_{k}^{2} I_{1}^{k} I_{1}^{k}}{\alpha_{n}^{2}-\alpha_{k}^{2}}, \\
n, k=1,2,3, k \neq n .
\end{array}\right\}
\end{aligned}
$$

Inserting the above values of the $\beta$ 's and c's coefficients into (74) and (75) and rearranging the terms so that the vectors $\pi^{0}, \pi^{1}, \hat{\mathbf{k}}$, and $\mathbf{r}$ are explicitly indicated, we obtain the final form of the first-order field as

$$
\begin{aligned}
\boldsymbol{\Phi}_{1}(\mathbf{r})= & \pi^{0} \cdot \tilde{A_{1}}(\rho)+\pi^{1} \otimes \hat{\mathbf{k}}: \tilde{\tilde{B}}_{1}(\rho) \cdot \mathbf{r} \\
& +\left(\rho^{2}-\alpha_{1}^{2}\right) \mathbf{R}^{e}\left[\pi^{0} \cdot \tilde{A}_{2}(\rho) \cdot \mathbf{r}+\pi^{1} \otimes \hat{\mathbf{k}}: \tilde{A}_{3}(\rho)+\pi^{1} \otimes \hat{\mathbf{k}}: \tilde{\tilde{B}}_{2}(\rho): \mathbf{r} \otimes \mathbf{r}\right],
\end{aligned}
$$

where the $\tilde{A}$ 's are second rank tensors, while the $\tilde{\tilde{B}}$ 's are fourth rank tensors given by

$$
\begin{aligned}
& \tilde{A_{1}}(\rho)=\frac{2\left(\tau^{3}+2\right)}{3 \tau} \sum_{n=1}^{3}\left(1-\frac{L_{0}^{n}(\rho)}{L_{n}^{0}}\right) \frac{\hat{\mathbf{x}}_{n} \otimes \hat{\mathbf{x}}_{n}}{L_{n}^{0}}, \\
& \tilde{A}_{2}(\rho)=-\frac{2\left(\tau^{3}+2\right)\left(\tau^{2}-1\right)}{3 \tau} \sum_{n=1}^{3} \frac{\hat{\mathbf{x}}_{n} \otimes \hat{\mathbf{x}}_{n}}{\left(\rho^{2}-\alpha_{1}^{2}+\alpha_{n}^{2}\right)\left(L_{0}^{n}\right)^{2}}, \\
& \tilde{A}_{3}(\rho)=\frac{\tau^{2}-1}{6\left(\Lambda-\Lambda^{\prime}\right)} \sum_{n=1}^{3} \tilde{\Gamma}_{n}\left[\frac{\left(\Lambda-\alpha_{1}^{2}\right)\left(\Lambda-\alpha_{2}^{2}\right)\left(\Lambda-\alpha_{3}^{2}\right)}{\Lambda-\alpha_{n}^{2}} \frac{1}{\left(\rho^{2}-\alpha_{1}^{2}+\Lambda\right)^{2}}\right. \\
& \left.-\frac{\left(\Lambda^{\prime}-\alpha_{1}^{2}\right)\left(\Lambda^{\prime}-\alpha_{2}^{2}\right)\left(\Lambda^{\prime}-\alpha_{3}^{2}\right)}{\left(\Lambda^{\prime}-\alpha_{n}^{2}\right)} \frac{1}{\left(\rho^{2}-\alpha_{1}^{2}+\Lambda^{\prime}\right)^{2}}\right] \text {, } \\
& \tilde{\tilde{B}}_{1}(\rho)=-\sum_{n=1}^{3} \sum_{\substack{k=1 \\
k \neq n}}^{3} \frac{1}{\Delta_{n k}}\left[\left(\tau^{2}-1\right)\left(I_{1}^{n} L_{1}^{n k}(\rho)-I_{1}^{n}(\rho) L_{1}^{n k}\right) \hat{\mathbf{x}}_{k} \otimes \hat{\mathbf{x}}_{n} \otimes \hat{\mathbf{x}}_{k} \otimes \hat{\mathbf{x}}_{n}\right. \\
& \left.+\left(\left(\tau^{2}+1\right) I_{1}^{k} I_{1}^{n}(\rho)+\left(\tau^{2}-1\right) \frac{\alpha_{n}^{2} I_{1}^{n} I_{1}^{n}(\rho)-\alpha_{k}^{2} I_{1}^{k} I_{1}^{k}(\rho)}{\alpha_{n}^{2}-\alpha_{k}^{2}}\right) \hat{\mathbf{x}}_{n} \otimes \hat{\mathbf{x}}_{k} \otimes \hat{\mathbf{x}}_{k} \otimes \hat{\mathbf{x}}_{n}\right], \\
& +\frac{\tau^{2}-1}{3} \sum_{n=1}^{3} \sum_{\substack{k=1 \\
k \neq n}}^{3} M_{n k}(\rho) \tilde{\Gamma}_{n} \otimes \hat{\mathbf{x}}_{k} \otimes \hat{\mathbf{x}}_{k} \\
& +\tilde{\mathrm{I}} \otimes \tilde{\mathrm{I}}+\tau^{2} \sum_{n=1}^{3} I_{1}^{n}(\rho) \tilde{\Gamma}_{n} \otimes \hat{\mathbf{x}}_{n} \otimes \hat{\mathbf{x}}_{n},
\end{aligned}
$$




$$
\begin{aligned}
& \tilde{\tilde{B}}_{2}(\rho)= \frac{\tau^{2}-1}{6\left(\Lambda-\Lambda^{\prime}\right)} \sum_{n=1}^{3} \sum_{k=1}^{3}\left[\frac{\left(\Lambda-\alpha_{1}^{2}\right)\left(\Lambda-\alpha_{2}^{2}\right)\left(\Lambda-\alpha_{3}^{2}\right)}{\left(\rho^{2}-\alpha_{1}^{2}+\Lambda\right)^{2}\left(\Lambda-\alpha_{n}^{2}\right)\left(\Lambda-\alpha_{k}^{2}\right)}\right. \\
&\left.-\frac{\left(\Lambda^{\prime}-\alpha_{1}^{2}\right)\left(\Lambda^{\prime}-\alpha_{2}^{2}\right)\left(\Lambda^{\prime}-\alpha_{3}^{2}\right)}{\left(\rho^{2}-\alpha_{1}^{2}+\Lambda^{\prime}\right)^{2}\left(\Lambda^{\prime}-\alpha_{n}^{2}\right)\left(\Lambda^{\prime}-\alpha_{k}^{2}\right)}\right] \tilde{\Gamma}_{n} \otimes \hat{\mathbf{x}}_{n} \otimes \hat{\mathbf{x}}_{n} \\
&+\left(\tau^{2}-1\right) \sum_{n=1}^{3} \sum_{\substack{k=1 \\
k \neq n}}^{3} \frac{I_{1}^{k}}{\left(\rho^{2}-\alpha_{1}^{2}+\alpha_{n}^{2}\right)\left(\rho^{2}-\alpha_{1}^{2}+\alpha_{k}^{2}\right) \Delta_{n k}} \hat{\mathbf{x}}_{n} \otimes \hat{\mathbf{x}}_{h} \otimes \hat{\mathbf{x}}_{n} .
\end{aligned}
$$

The tensors $\tilde{A}_{1}, \tilde{A}_{2}, \tilde{A}_{3}, \tilde{\tilde{B}}_{1}$, and $\tilde{\tilde{B}}_{2}$ are expressed in terms of the variable $\rho$, the parameter $\tau$ that controls the physics of the problem and the parameters $\alpha_{1}, \alpha_{2}, \alpha_{3}$ that describe the geometry of the scatterer.

6. The scattering amplitudes and cross section. Knowing the exact forms of the first two low-frequency fields it is possible to evaluate the normalized spherical scattering amplitudes up to the term $k^{2}$ by using the expansions (32)-(35)

$$
\begin{aligned}
& g_{r}(\hat{\mathbf{r}}, \hat{\mathbf{k}})=\frac{1}{c_{p}^{2}} \hat{\mathbf{r}} \cdot\left[k \mathbf{G}_{0,0}+k^{2}\left(\mathbf{G}_{1,0}+\mathbf{G}_{1,1}\right)\right]+O\left(k^{3}\right), \\
& g_{\vartheta}(\hat{\mathbf{r}}, \hat{\mathbf{k}})=\frac{1}{c_{s}^{2}} \hat{\boldsymbol{\vartheta}} \cdot\left[k \frac{\mathbf{G}_{0,0}}{\tau}+k^{2}\left(\frac{\mathbf{G}_{1,0}}{\tau}+\frac{\mathbf{G}_{1,1}}{\tau^{2}}\right)\right]+O\left(k^{3}\right), \\
& g_{\varphi}(\hat{\mathbf{r}}, \hat{\mathbf{k}})=\frac{1}{c_{s}^{2}} \hat{\boldsymbol{\varphi}} \cdot\left[k \frac{\mathbf{G}_{0,0}}{\tau}+k^{2}\left(\frac{\mathbf{G}_{1,0}}{\tau}+\frac{\mathbf{G}_{1,1}}{\tau^{2}}\right)\right]+O\left(k^{3}\right),
\end{aligned}
$$

as $k \rightarrow 0$, where

$$
\begin{aligned}
\mathbf{G}_{0,0} & =-\frac{i \tau}{4 \pi} \int_{S} T_{\mathbf{r}^{\prime}} \boldsymbol{\Phi}_{0}\left(\mathbf{r}^{\prime}\right) d s\left(\mathbf{r}^{\prime}\right), \\
\mathbf{G}_{1,0} & =\frac{\tau^{2}}{4 \pi} \int_{S} T_{\mathbf{r}^{\prime}} \boldsymbol{\Phi}_{1}\left(\mathbf{r}^{\prime}\right) d s\left(\mathbf{r}^{\prime}\right), \\
\mathbf{G}_{1,1} & =-\frac{\tau^{2}}{4 \pi} \int_{S} T_{\mathbf{r}^{\prime}} \boldsymbol{\Phi}_{0}\left(\mathbf{r}^{\prime}\right)\left(\hat{\mathbf{r}} \cdot \mathbf{r}^{\prime}\right) d s\left(\mathbf{r}^{\prime}\right) .
\end{aligned}
$$

The integral in (110) is known from the evaluation of the particular solution $\mathbf{P}_{1}(\mathbf{r})$. In particular, from (72) and (79) we obtain

$$
\mathbf{G}_{0,0}=2 i \tau \bar{\mu} \sum_{n=1}^{3} \frac{\pi_{n}^{0}}{L_{0}^{n}} \hat{\mathbf{x}}_{n} .
$$

The orthogonality of the surface ellipsoidal harmonics with respect to the weighting function $\left(\alpha_{1}^{2}-\mu^{2}\right)^{-1 / 2}\left(\alpha_{1}^{2}-\nu^{2}\right)^{-1 / 2}$ combined with the expression (77) implies that

$$
\mathbf{G}_{1,1}=\mathbf{0} \text {. }
$$


Very long calculations are needed in order to evaluate $T \Phi_{1}(\mathbf{r})$ on the surface of the scatterer, where

$$
T=2 \bar{\mu} \hat{\rho} \cdot \nabla+\lambda \hat{\rho} \operatorname{div}+\bar{\mu} \hat{\rho} \times \operatorname{rot}
$$

and $\boldsymbol{\Phi}_{1}(\mathbf{r})$ is given by (101). The integration of $T \boldsymbol{\Phi}_{1}$ over the surface of the scatterer is reduced to the following integrals which we evaluated by using ellipsoidal-spherical coordinates [10]

$$
\begin{gathered}
\int_{S} \mathbf{r} d s=\int_{S} \frac{\partial \mathbf{r}}{\partial \rho} d s=\int_{S} \mathbf{R}^{e} d s \\
=\int_{S} \hat{\boldsymbol{\rho}} d s=\int_{S} \frac{\hat{\boldsymbol{\rho}}}{\left(\alpha_{1}^{2}-\mu^{2}\right)\left(\boldsymbol{\alpha}_{1}^{2}-\nu^{2}\right)} d s=\mathbf{0}, \\
\int_{S} \frac{\mathbf{r} \otimes \mathbf{r} \otimes \hat{\boldsymbol{\rho}}}{\left(\alpha_{1}^{2}-\mu^{2}\right)\left(\alpha_{1}^{2}-\nu^{2}\right)} d s=\int_{S} \frac{\mathbf{r} \otimes \hat{\boldsymbol{\rho}} \otimes \hat{\boldsymbol{\rho}}}{\sqrt{\boldsymbol{\alpha}_{1}^{2}-\mu^{2}} \sqrt{\alpha_{1}^{2}-\nu^{2}}} d s=\mathbf{0} \otimes \mathbf{0} \otimes \mathbf{0}, \\
\int_{S} \frac{\mathbf{r} \otimes \hat{\boldsymbol{\rho}}}{\left(\alpha_{1}^{2}-\mu^{2}\right)\left(\alpha_{1}^{2}-\nu^{2}\right)} d s=4 \pi \sum_{n=1}^{3} \alpha_{n}^{2} I_{1}^{n} \hat{\mathbf{x}}_{n} \otimes \hat{\mathbf{x}}_{n}, \\
\int_{S} \frac{\hat{\boldsymbol{\rho}} \otimes \hat{\boldsymbol{\rho}}}{\sqrt{\boldsymbol{\alpha}_{1}^{2}-\mu^{2}} \sqrt{\boldsymbol{\alpha}_{1}^{2}-\nu^{2}}} d s=4 \pi \alpha_{1} \alpha_{2} \alpha_{3} \sum_{n=1}^{3} I_{1}^{n} \hat{\mathbf{x}}_{n} \otimes \hat{\mathbf{x}}_{n} .
\end{gathered}
$$

Using (116)-(119) we finally obtain

$$
\int_{S} T_{\mathbf{r}^{\prime}} \boldsymbol{\Phi}_{1}\left(\mathbf{r}^{\prime}\right) d s\left(\mathbf{r}^{\prime}\right)=-\frac{16 \pi \bar{\mu}\left(\tau^{3}+2\right)}{3 \tau} \sum_{n=1}^{3} \frac{\pi_{n}^{0}}{\left(L_{0}^{n}\right)^{2}} \hat{\mathbf{x}}_{n},
$$

which implies that

$$
\mathbf{G}_{1,0}=-\frac{4 \tau \bar{\mu}\left(\tau^{3}+2\right)}{3} \sum_{n=1}^{3} \frac{\pi_{n}^{0}}{\left(L_{0}^{n}\right)^{2}} \hat{\mathbf{x}}_{n}
$$

Substitution of (113), (114), and (121) into (107)-(109) yields the following expressions for the normalized spherical scattering amplitudes, as $k \rightarrow 0$ :

$$
\begin{aligned}
& g_{r}(\hat{\mathbf{r}}, \hat{\mathbf{k}})=\tau^{3} \hat{\mathbf{r}} \cdot \Lambda+O\left(k^{3}\right) \\
& g_{\theta}(\hat{\mathbf{r}}, \hat{\mathbf{k}})=\hat{\boldsymbol{\vartheta}} \cdot \Lambda+O\left(k^{3}\right) \\
& g_{\varphi}(\hat{\mathbf{r}}, \hat{\mathbf{k}})=\hat{\varphi} \cdot \Lambda+O\left(k^{3}\right)
\end{aligned}
$$

where

$$
\Lambda=\sum_{n=1}^{3}\left[2 i k-\frac{4\left(\tau^{3}+2\right)}{3 L_{0}^{n}} k^{2}\right] \frac{\pi_{n}^{0}}{L_{0}^{n}} \hat{\mathbf{x}}_{n}
$$


For the evaluation of the scattering cross section we substitute $g_{r}, g_{\theta}$, and $g_{\varphi}$ into (20) and (21) to obtain

$$
\begin{aligned}
\int_{|\hat{\mathbf{r}}|=1} & {\left[\frac{\left|g_{r}\right|^{2}}{k_{p}^{3}}+\frac{\left|g_{\theta}\right|^{2}+\left|g_{\phi}\right|^{2}}{k_{s}^{3}}\right] d s } \\
\quad= & \int_{|\hat{\mathbf{r}}|=1}\left[\frac{\tau^{6}}{k_{p}^{3}} \Lambda_{1} \cdot \hat{\mathbf{r}} \otimes \hat{\mathbf{r}} \cdot \Lambda_{1}^{*}+\frac{1}{k_{s}^{3}} \Lambda_{1} \cdot(\tilde{\mathbf{I}}-\hat{\mathbf{r}} \otimes \hat{\mathbf{r}}) \cdot \Lambda_{1}^{*}\right] d s+O\left(k^{2}\right), \\
& =\frac{1}{k_{s}^{3}} \int_{|\hat{\mathbf{r}}|=1}\left[\left|\Lambda_{1}\right|^{2}+\left(\tau^{3}-1\right) \Lambda_{1} \cdot \hat{\mathbf{r}} \otimes \hat{\mathbf{r}} \cdot \Lambda_{1}^{*}\right] d s+O\left(k^{2}\right) \\
& =\frac{4 \pi}{k_{s}^{3}} \frac{\tau^{3}+2}{3}\left|\Lambda_{1}\right|^{2}+O\left(k^{2}\right),
\end{aligned}
$$

where

$$
\Lambda_{1}=2 i k \sum_{n=1}^{3} \frac{\pi_{n}^{0}}{L_{0}^{n}} \hat{\mathbf{x}}_{n} .
$$

Therefore, for $P$-incidence we obtain

$$
\sigma^{p}=\frac{16 \pi}{3} \tau\left(\tau^{3}+2\right) \sum_{n=1}^{3}\left(\frac{i_{n}}{L_{0}^{n}}\right)^{2}+O\left(k^{2}\right),
$$

while for $S$-incidence

$$
\sigma^{s}=\frac{16 \pi}{3}\left(\tau^{3}+2\right) \sum_{n=1}^{3}\left(\frac{b_{n}}{L_{0}^{n}}\right)^{2}+O\left(k^{2}\right) .
$$

We observe that the leading term of the scattering cross section is independent of the wave number, while the difference between $\sigma^{p}$ and $\sigma^{s}$ (besides the components $i_{n}$ and $b_{n}$ of the polarization vectors) is the factor $\tau$.

\section{Geometrically degenerate cases.}

A. Spheroids. A prolate spheroid is obtained whenever $\alpha_{1}>\alpha_{2}=\alpha_{3}$ while the case of an oblate spheroid corresponds to $\alpha_{1}<\alpha_{2}=\alpha_{3}$.

The elliptic integrals can be evaluated in closed form for spheroids

$$
\begin{aligned}
& I_{0}^{1}(\rho)=\frac{1}{h_{3}}\left\{\begin{array}{lc}
\frac{1}{2} \ln \left(\frac{\rho+h_{3}}{\rho-h_{3}}\right), & \alpha_{1}>\alpha_{2}, \\
\frac{1}{i} \tan ^{-1}\left(\frac{i h_{3}}{\rho}\right), & \alpha_{1}<\alpha_{2},
\end{array}\right. \\
& I_{1}^{1}(\rho)=\frac{1}{h_{3}^{2}}\left(I_{0}^{1}(\rho)-\frac{1}{\rho}\right), \\
& I_{1}^{2}(\rho)=I_{1}^{3}(\rho)=-\frac{1}{2 h_{3}^{2}}\left(I_{0}^{1}(\rho)-\frac{\rho}{\rho^{2}-h_{3}^{2}}\right),
\end{aligned}
$$




$$
\begin{aligned}
& I_{2}^{1}(\rho)=\frac{9}{4 h_{3}^{4}}\left(I_{0}^{1}(\rho)-\frac{3 \rho}{3 \rho^{2}-h_{3}^{2}}\right), \\
& I_{2}^{2}(\rho)=I_{2}^{5}(\rho)=\frac{3}{8 h_{3}^{4}}\left(I_{0}^{1}(\rho)-\frac{\rho\left(3 \rho^{2}-5 h_{3}^{2}\right)}{3\left(\rho^{2}-h_{3}^{2}\right)^{2}}\right), \\
& I_{2}^{3}(\rho)=I_{2}^{4}(\rho)=-\frac{3}{2 h_{3}^{4}}\left(I_{0}^{1}(\rho)-\frac{3 \rho^{2}-2 h_{3}^{2}}{3 \rho\left(\rho^{2}-h_{3}^{2}\right)}\right),
\end{aligned}
$$

where

$$
\rho=h_{3}\left\{\begin{array}{l}
\cosh \omega \\
i \sinh \omega
\end{array}= \begin{cases}\sqrt{\alpha_{1}^{2}-\alpha_{2}^{2}} \cosh \omega, & \alpha_{1}>\alpha_{2}, \\
\sqrt{\alpha_{2}^{2}-\alpha_{1}^{2}} \sinh \omega, & \alpha_{1}<\alpha_{2},\end{cases}\right.
$$

and $(\omega, \vartheta, \varphi)$ are the spheroidal coordinates which are related to the Cartesian coordinates $\left(x_{1}, x_{2}, x_{3}\right)$ by

$$
\begin{aligned}
& x_{1}=\rho \cos \vartheta, \quad \omega \in[0,+\infty), \\
& x_{2}=\sqrt{\rho^{2}-h_{3}^{2}} \sin \vartheta \cos \varphi, \quad \vartheta \in[0, \pi], \\
& x_{3}=\sqrt{\rho^{2}-h_{3}^{2}} \sin \vartheta \sin \varphi, \quad \varphi \in[0,2 \pi) .
\end{aligned}
$$

For $\rho=\alpha_{1}$, we obtain

$$
I_{0}^{1}=\frac{1}{\alpha_{2}} \begin{cases}{\left[\left(\frac{\alpha_{1}}{\alpha_{2}}\right)^{2}-1\right]^{-1 / 2} \cosh ^{-1}\left(\frac{\alpha_{1}}{\alpha_{2}}\right),} & \alpha_{1}>\alpha_{2}, \\ {\left[1-\left(\frac{\alpha_{1}}{\alpha_{2}}\right)^{2}\right]^{-1 / 2} \cos ^{-1}\left(\frac{\alpha_{1}}{\alpha_{2}}\right),} & \alpha_{1}<\alpha_{2},\end{cases}
$$

and through (130)-(134) all the other elliptic integrals can be expressed as functions of the ratio $\alpha_{1} / \alpha_{2}$, whenever $\rho=\alpha_{1}$.

Having the values of the elliptic integrals we can substitute them in the corresponding expressions and obtain the results for an oblate or a prolate spheroid, as the case may be.

B. Needle and disc. The needle-shaped scatterer can be approximated by a prolate spheroid where $\alpha_{1} \gg \alpha_{2}=\alpha_{3}$. In this case

$$
I_{0}^{1} \sim \frac{1}{\alpha_{2}} \frac{\ln 2\left(\alpha_{1} / \alpha_{2}\right)}{\left(\alpha_{1} / \alpha_{2}\right)}, \quad \frac{\alpha_{1}}{\alpha_{2}} \rightarrow+\infty .
$$

In the case where $\alpha_{1} \ll \alpha_{2}=\alpha_{3}$, the oblate spheroid takes the shape of a circular disc and

$$
I_{0}^{1} \sim \frac{\pi}{2 \alpha_{2}}, \quad \frac{\alpha_{1}}{\alpha_{2}} \rightarrow 0+.
$$

C. Sphere. The sphere is the shape that corresponds to radial symmetry and comes out of the case where $\alpha_{1}=\alpha_{2}=\alpha_{3}=\alpha$. 
The elliptic integrals assume the following values:

$$
\begin{gathered}
I_{0}^{1}(\rho)=1 / \rho, \\
I_{1}^{n}(\rho)=1 / 3 \rho^{3}, \quad n=1,2,3, \\
I_{2}^{n}(\rho)=1 / 5 \rho^{5}, \quad n=1,2,3,4,5 .
\end{gathered}
$$

We also obtain that $\rho=r, \mu=\nu=0$, and $\Lambda=\Lambda^{\prime}=\alpha^{2}$. In order to evaluate the undetermined forms in the various expressions it is enough to approximate the sphere, say by a prolate spheroid setting $\alpha_{1}=\alpha(1+\varepsilon), \varepsilon>0, \alpha_{2}=\alpha_{3}=\alpha$, and obtain the case of a sphere in the limit as $\varepsilon \rightarrow 0+$.

8. Discussion. The physical setting of this work is described by a plane harmonic elastic wave which propagates on an isotropic and homogeneous medium containing a rigid inhomogeneity in the shape of a general triaxial ellipsoid. The incident plane wave is either a transverse or a longitudinal wave with a propagation vector that is arbitrarily oriented with respect to the principal axes of the ellipsoid. The wavelenth of the incident wave is supposed to be much smaller than the largest axis of the scatterer. Due to the mode conversion on the boundary of the ellipsoidal discontinuity of the medium, both longitudinal and transverse waves will be radiated from the scatterer.

The zeroth-order approximation $\boldsymbol{\Phi}_{0}$ of the total field is given by (68) and it consists of two terms. The first term in the right-hand side of (68) is characterized as the Cartesian part of $\boldsymbol{\Phi}_{0}$, while the second term in (68) is characterized as the ellipsoidal part and it is directed along the outward unit normal on the surface of the ellipsoid. Due to the factor $\left(\rho^{2}-a_{1}^{2}\right)$, the ellipsoidal part of $\boldsymbol{\Phi}_{0}$ vanishes on the surface of the scatterer. The ellipsoidal part of $\Phi_{0}$ is also proportional to the factor $1-\tau^{2}$. The parameter $\tau^{2}$ determines the ratio of the phase velocity of the transverse to the phase velocity of the longitudinal wave. The smaller the sum $\lambda+\bar{\mu}$ of the Lamé constants, the closer to zero the value of $1-\tau^{2}$ is and hence the less prominent the ellipsoidal part of $\Phi_{0}$. The case of $\lambda+\bar{\mu}=0$ reduces the elastostatic equation to the Laplace equation and the whole theory of low-frequency elastic scattering to the corresponding theory for acoustic scattering $[2,3,4,5]$. It is of interest to note that the propagation vector $\hat{\mathbf{k}}$ does not enter the expression for $\boldsymbol{\Phi}_{0}$ which is dependent on the polarization vector $\pi^{0}$.

For the first-order approximation $\Phi_{1}$, which is given by (101), we observe that there exist again a Cartesian and an ellipsoidal part. The field $\boldsymbol{\Phi}_{1}$ involves the second rank tensors $\tilde{A}_{1}, \tilde{A}_{2}, \tilde{A}_{3}$ and the fourth rank tensors $\tilde{\tilde{B}}_{1}, \tilde{\tilde{B}}_{2}$. All these tensors are given by very complicated expressions of the following variables: the variable $\rho$ that corresponds to the radial variable $r$ of the spherical coordinates and is a measure of the distance of the observation point $\mathbf{r}$ from the scatterer, the parameter $\tau^{2}$ that determines the ratio of the two speeds of propagation, and the three semiaxes $a_{1}, a_{2}, a_{3}$ of the ellipsoid. The parameter $\tau^{2}$ describes the physical properties of the medium through the values of the Lamé constants $\lambda$ and $\bar{\mu}$. On the other hand the values of $a_{1}, a_{2}, a_{3}$ describe the geometrical characteristics of the scattering region. It is the variation of these semiaxes that provides us with the corresponding solutions to the problem of the sphere whenever $a_{1}=a_{2}=a_{3}$, the prolate spheroid whenever $a_{1}>a_{2}=a_{3}$, the oblate spheroid whenever 
$a_{1}=a_{2}>a_{3}$, the needle as the extreme case of a prolate spheroid, or the disc as the extreme case of an oblate spheroid. As it is readily observed from (101), the actual value of $\Phi_{1}$ is obtained by contracting the tensors $\tilde{A}$ and $\tilde{\tilde{B}}$ by the polarization and propagation vectors as well as by the observation vector. The tensors $\tilde{A}_{2}, \tilde{A}_{3}$, and $\tilde{\tilde{B}}_{2}$ are proportional to the factor $1-\tau^{2}$. Hence, as in the case of $\Phi_{0}$, the ellipsoidal part of $\Phi_{1}$ weakens whenever the phase velocity of the longitudinal wave does not differ much from the phase velocity of the transverse wave. Similarly the ellipsoidal part of $\Phi_{1}$ vanishes on the surface of the scatterer due to the $\rho^{2}-a_{1}^{2}$ factor. The Cartesian part of $\Phi_{1}$ involves a term that is independent of $\mathbf{r}$ and a term directly dependent upon $\mathbf{r}$. The ellipsoidal part involves a term independent of $\mathbf{r}$, a term dependent upon $\mathbf{r}$ and finally a term containing $\mathbf{r} \otimes \mathbf{r}$.

The scattering cross section which measures the total energy that the scatterer takes from the incident wave and reradiates as a scattered field determines the global effect of the scattering region on the propagation of the incident wave. It is given by (20) for longitudinal and by (21) for transverse incidence, in terms of the normalized (dimensionless) spherical scattering amplitudes $g_{r}, g_{\vartheta}, g_{\varphi}$. Since the wave number $k_{p}$ for the longitudinal wave is less than the wave number for the transverse wave $k_{s}$, it follows that the contribution of the radial scattering amplitude $g_{r}$ to the scattering cross section is larger than the contribution of each one of the two angular scattering amplitudes $g_{\vartheta}$ and $g_{q}$. This is in accordance with the intuitive physical reasoning that in the far-field the scattered wave propagates mainly as a radially expanding spherical wave.

In the low-frequency theory the normalized spherical scattering amplitudes are given by (32), (33), and (34) as power series of $k$. The coefficients of these expansions involve the integrals that appear in (35), which are nothing else but moments of the low-frequency approximations of the traction field on the surface of the scatterer. As it is easily seen in (107)-(109), the leading terms of the low-frequency expansions of $g_{r}, g_{\vartheta}$, and $g_{\varphi}$ are of the order $k$ and they are all proportional to the zeroth-order moment of the traction of $\boldsymbol{\Phi}_{0}$ on the surface of the scatterer, which for the case of the ellipsoid is the constant vector given in (113). The constant vector $\mathbf{G}_{0.0}$ given by (113) forms the leading approximation to the general scattering amplitude and it is analyzed on the $\hat{\mathbf{r}}, \hat{\boldsymbol{\theta}}, \hat{\varphi}$ directions with corresponding weights $1 / c_{p}^{2}, 1 / c_{s}^{2} \tau, 1 / c_{s}^{2} \tau$ which determine the distribution of $\mathbf{G}_{0,0}$ into the spherical scattering amplitudes $g_{r}, g_{\vartheta}, g_{\varphi}$.

Formulae (127) and (128) give the first approximation of the scattering cross section for longitudinal and for transverse incidence, respectively. They are both constants independent of the wave number $k$. Besides the components of the polarization vectors $\hat{\mathbf{k}}$ and $\hat{\mathbf{b}}$, they only differ by a factor $\tau$. This implies that, as far as the first low-frequency approximation is concerned, a rigid ellipsoid scatters more energy from a transverse rather than from a longitudinal incident wave and this difference becomes larger as the difference of the two phase velocities becomes larger.

Finally, since $a_{1}>a_{2}>a_{3}>0$, we obtain that

$$
0<\frac{1}{\left(L_{0}^{1}\right)^{2}}<\frac{1}{\left(L_{0}^{2}\right)^{2}}<\frac{1}{\left(L_{0}^{3}\right)^{2}},
$$

which implies that the largest value of the first term for the scattering cross section occurs for incident polarization parallel to the smallest semiaxis of the ellipsoid. For longitudinal 
incidence the maximum value of the leading term of $\sigma^{p}$ requires direction of incidence parallel to the $x_{3}$-axis, while for transverse incidence it requires a direction of incidence on the $x_{1} x_{2}$-plane. Similarly the minimum value of the first term of $\sigma$ is obtained whenever the polarization vector is parallel to the largest semiaxis $\alpha_{1}$.

All the results of this paper are expressed in a form suitable for physical investigations as well as numerical calculations which are actually reduced to numerical values of given functions. Besides that, many interesting calculations for the special shapes of the ellipsoid can be performed in closed analytical form. As an example, we give the value of the leading low-frequency approximation for the scattering cross section whenever a longitudinal wave is scattered by a rigid sphere of radius $a$. In this case formulae (127), (140), and (67) give

$$
\sigma^{p}=12 \pi a^{2} \frac{\tau^{4}+2 \tau}{\tau^{4}+4 \tau^{2}+4},
$$

which implies that the total energy scattered by a rigid sphere is equal to the area of a large circle of the sphere times a constant which depends solely on the ratio of the phase velocity of the transverse to the longitudinal wave.

\section{REFERENCES}

[1] P. J. Barratt and W. D. Collins, The scattering cross section of an obstacle in an elastic solid for plane harmonic n'aves, Proc. Camb. Phil. Soc. 61, 969 (1965)

[2] (3. Dassios, Convergent low-frequency expansions for penetrable scatterers, Doctoral Dissertation, University of Illinois at Chicago Circle, 1975

[3] (j. Dassios, Convergent low-frequency expansions for penetrable scatterers, J. Math. Phys. 18, 126 (1977)

[4] (i. Dassios, Second order low-frequency scattering by the soft ellipsoid, SIAM J. Appl. Math. 38, 373 (1980)

[5] (3. Dassios, Low-frequency scattering theory for a penetrable hody with an impenetrable core, SIAM J. Appl. Math. 42, 272 (1982)

[6] G. Dassios, Scattering of acoustic waves by a coated pressure-release ellipsoid, J. Acoust. Soc. Am. 70, 176 (1981)

[7] (3. Dassios and K. Kiriaki, The low-frequency theory of elastic wave scattering, Quart. Appl. Math. 42, 225 (1984)

[8] (3. Dassios and K. Kiriaki, The ellipsoidal cavity in the presence of a low-frequency elastic wave, Quart. Appl. Math. (1987)

[9] N. G. Einspruch, E. J. Witterholt and R. Truell, Scattering of a plane transverse wave hy a spherical obstacle in an elastic medium, J. Appl. Phys. 31, 806 (1960)

[10] E. W. Hobson, The theory of spherical and ellipsoidal harmonics, Chelsea, 1955

[11] V. D. Kupradze, Dynamical problems in elasticity, Progress in solid mechanics III, North-Holland, 1963

[12] E. G. Lawrence, Diffraction of elastic waves by a rigid ellipsoid, Quart. J. Mech. Appl. Math. XXV, 161 (1972)

[13] L. Solomon, Elasticité linéaire, Masson et cie, 1968

[14] V. Twersky, Certain transmission and reflection theorems, J. Appl. Phys. 25, 859 (1954)

[15] C. Truesdell, Mechanics of solids II, Encyclopedia of Physics, Volume VI a/2, Springer-Verlag, 1972

[16] C. F. Ying and R. Truell, Scattering of a plane longitudinal wave by a spherical obstacle in an isotropically elastic solid. J. Appl. Phys. 27, 1086 (1956) 